\title{
Langmuir
}

pubs.acs.org/Langmuir

(C) 2009 American Chemical Society

\section{X-ray Photoelectron Spectroscopy- and Surface Plasmon Resonance-Detected Photo Release of Photolabile Protecting Groups from Nucleoside Self-Assembled Monolayers on Gold Surfaces}

\author{
Katja Drexler, ${ }^{\dagger}$ Julia Smirnova, ${ }^{\dagger}$ Marina Galetskaya, ${ }^{\dagger}$ Sönke Voss, ${ }^{\star}$ Mikhail Fonin, ${ }^{\star}$ \\ Johannes Boneberg, ${ }^{\star}$ Ulrich Rüdiger, ${ }^{\star}$ Paul Leiderer, ${ }^{\star}$ and Ulrich E. Steiner, ${ }^{*}$ \\ ${ }^{\dagger}$ Department of Chemistry and ${ }^{\star}$ Department of Physics, University of Konstanz, D-78457 Konstanz, Germany
}

Received April 16, 2009. Revised Manuscript Received June 28, 2009

\begin{abstract}
The formation of self-assembled monolayers (SAMs) on gold by 2-(5-iodo-2-nitrophenyl) propoxycarbonyl (I-NPPOC)-protected thymidine with an attached mercaptohexyl succinate linker and the kinetics of photochemical release of the I-NPPOC group were monitored using X-ray photoelectron spectroscopy (XPS) and surface plasmon resonance (SPR) detection. In the XPS spectra, the iodine peaks allowed for specific and accurate monitoring of the presence and loss of I-NPPOC groups on the surface. In the SPR experiment, the overall signal change on photoillumination is in accord with a theoretical estimation of the density of I-NPPOC groups in a dense monolayer. The kinetics roughly follow a biexponential time dependence with two very different time constants, corresponding to photochemical quantum yields of 0.22 and 0.0032 , respectively.
\end{abstract}

\section{Introduction}

DNA microarrays or DNA chips have been established as powerful tools for genomic studies in modern biotechnology. ${ }^{1,2}$ Arrays with more than 1 million $^{3}$ different oligonucleotide probes and the potential of mapping whole genomes have become accessible through photolithographic techniques. In the synthesis of such highdensity arrays, photoremovable protecting groups play a key role because they allow the controlled stepwise and spatially addressable assembly of nucleotides in an efficient manner. ${ }^{4}$ Since the quality of the chips and the efficiency of their production critically depend on the photochemical performance of the photoremovable protecting groups, there is considerable interest in optimizing their performance on a chip. ${ }^{5-9}$ Ideally, one would like to monitor and characterize their kinetic behavior under chip conditions by direct in situ methods without recourse to destructive monitoring methods where the process of chip synthesis is terminated, as is the case with reactive fluorescence labeling, today's standard method for assessing the

*To whom correspondence should be addressed. Fax: +49 7531883014 . E-mail: Ulrich.steiner@uni-konstanz.de.

(1) Nuwaysir, E. F.; Huang, W.; Albert, T. J.; Singh, J.; Nuwaysir, K.; Pitas, A.; Richmond, T.; Gorski, T.; Berg, J. P.; Ballin, J.; McCormick, M.; Norton, J.; Pollock, T.; Sumwalt, T.; Butcher, L.; Porter, D.; Molla, M.; Hall, C.; Blattner, F.; Sussman, M. R.; Wallace, R. L.; Cerrina, F.; Green, R. D. Genome Res. 2002, 12, 1749-1755.

(2) Wang, D. G.; Fan, J.-B.; Siao, C.-J.; Berno, A.; Young, P.; Sapolsky, R.; Ghandour, G.; Perkins, N.; Winchester, E.; Spencer, J.; Kruglyak, L.; Stein, L.; Hsie, L.; Topaloglou, T.; Hubbell, E.; Robinson, E.; Mittmann, M.; Morris, M. S.; Shen, N.; Kilburn, D.; Rioux, J.; Nusbaum, C.; Rozen, S.; Hudson, T. J.; Lipshutz, R.; Chee, M.; Lander, E. S. Science 1998, 280, 1077-1082.

(3) Gershon, D. Nature 2005, 437, 1195-1198.

(4) Pirrung, M. C.; Rana, V. S. Photoremovable Protecting Groups in DNA Synthesis and Microarray Fabrication. In Dynamic Studies in Biology: Phototriggers, Photoswitches, and Caged Compounds; Givens, R. S., Goeldner, M., Eds.; John Wiley \& Sons: New York, 2005; p 341

(5) Wöll, D.; Walbert, S.; Stengele, K.-P.; Albert, T. J.; Richmond, T.; Norton, J.; Singer, M.; Green, R. D.; Pfleiderer, W.; Steiner, U. E. Helv. Chim. Acta 2004, 87, $28-45$. (6) Smirnova, J.; Wöll, D.; Pfleiderer, W.; Steiner, U. E. Helv. Chim. Acta 2005, $88,891-904$

(7) Wöll, D.; Smirnova, J.; Pfleiderer, W.; Steiner, U. E. Angew. Chem. 2006, 45, 2975-2978.

(8) Wöll, D.; Laimgruber, S.; Galetskaya, M.; Smirnova, J.; Pfleiderer, W.; Heinz, B.; Gilch, P.; Steiner, U. E. J. Am. Chem. Soc. 2007, 129, 12148-12158.

(9) Wöll, D.; Smirnova, J.; Galetskaya, M.; Prykota, T.; Bühler, J.; Stengele, K P.; Pfleiderer, W.; Steiner, U. E. Chem.-Eur. J. 2008, 14, 6490-6497.

(10) McGall, G. H.; Barone, A. D.; Diggelsmann, M.; Fodor, S. P. A.; Gentalen, E.; Ngo, N. J. Am. Chem. Soc. 1997, 119, 5081-5090. photokinetics of protecting group release on chips. ${ }^{10}$ Surface plasmon resonance (SPR) spectroscopy has proven to be very successful for sensing and characterizing molecular recognition events at self-assembled monolayers (SAMs) on gold and silver surfaces. Effective refractive index changes at the interface, as, for example, caused by thickness changes in the adsorbed layer, lead to proportional changes in the resonance angle of surface plasmons at a given wavelength. ${ }^{11-16}$ Correspondingly, photoinduced changes in the surface adjacent layer are also expected to lead to detectable SPR signals. However, since electronically excited states in the vicinity of metal surfaces are liable to quenching by energy transfer to the metal, ${ }^{17}$ how efficient molecular photoreactions could occur in microscopic environments that are typical for SPR detection is a question of general interest. In fact, despite a possible electronic quenching by the metal, a number of photochemical changes in self-assembled monolayers (SAMs) on thin metal layers or metal nanoparticles have recently been reported. Most of these have utilized cis-trans photoisomerizations of functionalized azobenzenes or stilbenes; ${ }^{18-27}$ others involve

(11) Raether, H. Surface Plasmons on smooth and rough surfaces and on gratings; Springer-Verlag: Berlin, 1988; Vol. 111.

(12) Knoll, W. Anпu. Rev. Phys. Chem. 1998, 49, 569-638.

(13) Homola, J.; Yee, S. S.; Gauglitz, G. Sens. Actuators, B 1999, 54, 3-15.

(14) Jönsson, U.; Fagerstam, L.; Ivarson, B.; Johnsson, B.; Karlsson, R.; Lundh, K.; Lofas, S.; Persson, B.; Roos, H.; Ronnberg, I.; Sjölander, S.: Stenberg, E.; Ståhlberg, R.; Urbanicky, C.; Ostlin, H.; Malmqvist, M. BioTechniques 1991, $11,620-627$.

(15) Jung, L. S.; Campbell, C. T.; Chinowsky, T. M.; Mar, M. N.; Yee, S. S. Langmuir 1998, 14, 5636-5648.

(16) Geldhauser, T.; Leiderer, P.; Boneberg, J.; Walheim, S.; Schimmel, T. Langmuir 2008, 24, 13155-13160.

(17) Waldeck, D. H.; Alivisatos, A. P.; Harris, C. B. Surf. Sci. 1985, 158, 103-125.

(18) Sekkat, Z.: Wood, J.; Geerts, Y.; Knoll, W. Langmuir 1996, 12, 2976-2980.

(19) Tamada, K.; Akiyama, H.; Wei, T. X. Langmuir 2002, 18, 5239-5246.

(20) Tamada, K.; Akiyama, H.; Wei, T. X.; Kim, S. A. Langmuir 2003, 19, 2306 2312

(21) Wolf, M. O.; Fox, M. A. J. Am. Chem. Soc. 1995, 117, 1845-1846.

(22) Sortino, S.; Petralia, S.; Conoci, S.; Bella, S. D. J. Mater. Chem. 2004, 14, 811-813.

(23) Callari, F. L.; Sortino, S. J. Mater. Chem. 2007, 17, 4184-4188.

(24) Callari, F.; Petralia, S.; Sortino, S. Chem. Commun. 2006, 1009-1011.

(25) Manna, A.; Chen, P. L.; Akiyama, H.; Wei, T. X.; Tamada, K.; Knoll, W. Chem. Mater. 2003, 15, 20-28.

(26) Zhang, J.; Whitesell, J. K.; Fox, M. A. Chem. Mater. 2001, 13, 2323-2331.

(27) Wolf, M. O.; Fox, M. A. Langmuir 1996, 12, 955-962. 

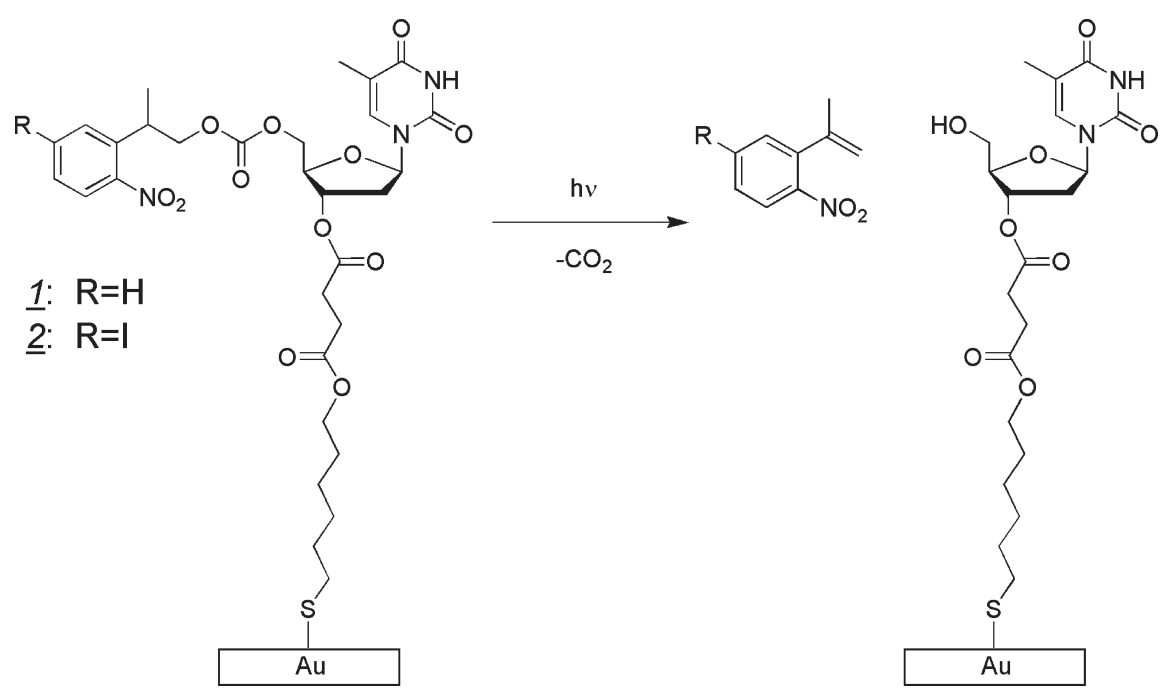

Figure 1. Photoreaction of $\mathbf{1}$ or $\mathbf{2}$ adsorbed on a gold surface.

photochemical ring opening of a spiropyran, ${ }^{28}$ photoinduced switching of a dithienylethane between ring closure and opening, ${ }^{29}$ photodimerization of coumarin, ${ }^{30}$ photocleavage of aromatic nitro compounds, ${ }^{31-33}$ or photo-defluorination. ${ }^{34}$ These photochemical changes have been monitored by FTIR, contact angle, fluorescence, and absorption measurements, ${ }^{21-27}$ and in several cases also by SPR. ${ }^{18-20,29}$

In this paper, we present the first example of selective photodeprotection of nucleoside SAMs on gold surfaces with in situ monitoring of the corresponding photokinetics by SPR. To prove that the photoinduced change in the SPR signal is in fact due to the photoinduced cleavage of the protecting group, independent experiments employing X-ray photoelectron spectroscopy (XPS) were performed in parallel. Since the photolabile protecting group was marked with an iodine substituent, a pronounced marker signal due to its large Scofield factor at the excitation energy was observed. ${ }^{35}$ Among other methods, XPS analysis has been occasionally applied in probing and characterizing the formation of SAMs, ${ }^{21,33,36-38}$ but rarely for the verification of photochemistry. ${ }^{34,39}$ In ref 39, an iodine-carbon bond was cleaved by short-wave UV light and the loss of iodine was detected by XPS. In our work, no direct iodine photochemistry is involved. The iodine function is restricted to representing a label of the photolabile protecting group.

3923

Kaganer, E.; Pogreb, R.; Davidov, D.; Willner, I. Langmuir 1999, 15, 3920-

(29) Masson, J. F.; Liddell, P. A.; Banerji, S.; Battaglia, T. M.; Gust, D.; Booksh, K. S. Langmuir 2005, 21, 7413-7420.

(30) Li, W.; Lynch, V.; Thompson, H.; Fox, M. A. J. Am. Chem. Soc. 1997, 119, $7211-7217$

(31) Hu, J.; Zhang, J.; Liu, F.; Kittredge, K.; Whitesell, J. K.; Fox, M. A. J. Am. Chem. Soc. 2001, 123, 1464-1470.

(32) Caruso, E. B.; Petralia, S.; Conoci, S.; Giuffrida, S.; Sortino, S. J. Am. Chem. Soc. 2007, 129, 480-481.

(33) Sortino, S.; Petralia, S.; Compagnini, G.; Conoci, S.; Condorelli, G. Angew. Chem., Int. Ed. 2002, 41, 1914-1917.

(34) Sortino, S.; Petralia, S.; Condorelli, G. G.; Conoci, S.; Condorelli, G. Langmuir 2003, 19, 536-539.

(35) Scofield, J. H. J. Electron Spectrosc. Relat. Phenom. 1976, 8, 129-137.

(36) Castner, D. G.; Hinds, K.; Grainger, D. W. Langmuir 1996, 12, 5083-5086.

(37) Nelson, K. E.; Gamble, L.; Jung, L. S.; Boeck1, M. S.; Naeemi, E.; Golledge, S. L.; Sasaki, T.; Castner, D. G.; Campbell, C. T.; Stayton, P. S. Langmuir 2001, 17, 2807-2816.

(38) Sortino, S.; Petralia, S.; Conoci, S.; Bella, S. D. J. Am. Chem. Soc. 2003, 125, $1122-1123$.

(39) Siffalovic, P.; Michelswirth, M.; Bartz, P.; Decker, B.; Agena, C.; Schäfer, C.; Molter, S.; Ros, R.; Bach, M.; Neumann, M.; Anselmetti, D.; Mattay, J.; Heinzmann, U.; Drescher, M. J. Biotechnol. 2004, 112, 139-149.

\section{Experimental Section}

Samples. The photolabile compounds $5^{\prime}$-O-[2-(2-nitrophenyl)propoxycarbonyl]-3'-O-[3-(6-mercaptohexyloxycarbonyl)propanoyl]thymidine (1), its 5-iodo derivative (2) (cf. Figure 1), and the nonphotolabile reference compound 6-(4-iodobenzyloxy)hexane-1-thiol (3) were synthesized as described in the Supporting Information.<smiles>O=COCc1ccc(I)cc1</smiles>

$\underline{3}$

XPS Measurements. XPS measurements were performed in an Omicron Multiprobe ultra-high vacuum system (base pressure of $10^{-11}$ mbar) with $\mathrm{Al} \mathrm{K} \alpha$ radiation $(h v=1486.6 \mathrm{eV})$ from a dual-anode X-ray source. The energy resolution of the EA 125 analyzer was set to $0.65 \mathrm{eV}$. The background-corrected integral iodine $3 \mathrm{~d}$ peak intensity was determined with EIS (Omicron Nanotechnology GmbH).

SAM Preparation for XPS. The substrates were cut from a gold-coated silicon wafer [Aldrich, Au layer thickness of $1000 \AA$, $99.999 \%$ pure, with a titanium (99.9\% pure) adhesion layer between the gold and silicon wafer] in pieces of $5 \mathrm{~mm} \times 10 \mathrm{~mm}$. The gold substrates were cleaned with acetone and dried in a stream of nitrogen. For adsorption of the SAM forming thiol 2 , the substrates were placed in a $1 \mathrm{mM}$ ethanolic solution for $1 \mathrm{~h}$. Tests with various adsorption times had ensured that the adsorption process had definitely reached a saturation limit after such a time. After being washed repeatedly with ethanol, the substrates were dried in a stream of nitrogen.

Illumination Equipment. A 200 W UV light source(Lumatec, Superlite SUV-DC-P) with a liquid light guide (Lumatec series 300 , diameter of $3 \mathrm{~mm}$, length of $1500 \mathrm{~mm}$, N.A. $=2 \alpha=72^{\circ}$ ). The end of the light guide was coupled to a second liquid light guide by a unit containing a $365 \mathrm{~nm}$ interference filter between two collimation lenses. The output of $365 \mathrm{~nm}$ light at the second light guide amounted to typically $6-8 \times 10^{-8} \mathrm{E} \mathrm{s}^{-1}$ as measured by azobenzene actinometry. ${ }^{40,41}$

Illumination of XPS Samples. The samples were illuminated using liquid light guide optics (Lumatec, Series 300) with the free fiber tip ending $\sim 5-7 \mathrm{~mm}$ above the substrate. The light guide included a filter compartment, where an appropriate interference filter was inserted. Across the substrate, the illumination light flux

(40) Gauglitz, G.; Hubig, S. J. Photochem. 1981, 15, 256-257.

(41) Gauglitz, G. J. Photochem. 1976, 5, 41-47. 
a)

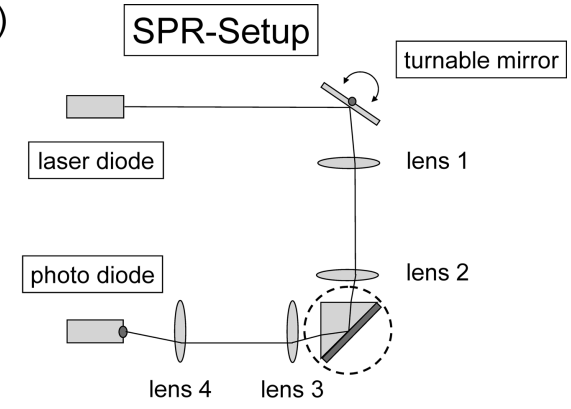

b)

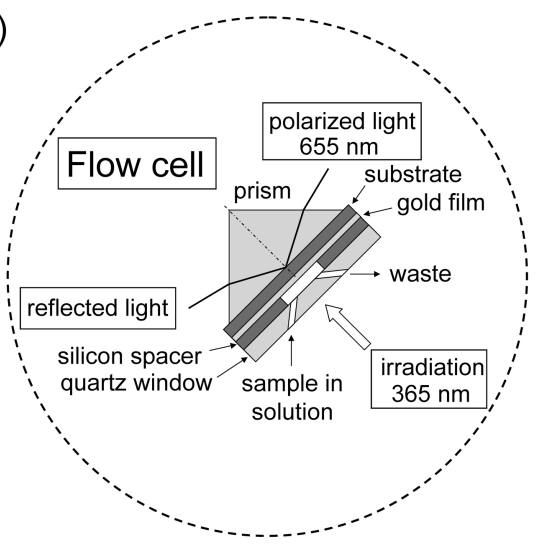

Figure 2. (a) Schematic of the experimental setup for SPR (b) Schematic of the flow cell.

density at $365 \mathrm{~nm}$ ranged between 0.5 and $1 \times 10^{-7} \mathrm{E} \mathrm{cm}^{-2} \mathrm{~s}^{-1}$ depending on the exact distance between the fiber tip and substrate. During illumination, the substrate surface was rinsed with a continuous stream of fresh absolute ethanol delivered through an injection needle at a rate of $0.5 \mathrm{~mL} / \mathrm{min}$ by a peristaltic pump (Ismatec).

Surface Plasmon Spectroscopy (SPR). The schematic setup for SPR is shown in Figure 2a. The beam of a laser diode (Single Mode, Sanyo, $60 \mathrm{~mW}, \lambda=658 \mathrm{~nm}$ ) hits a mirror, the angle of which is controlled by a stepper motor. The reflected beam passes through two lenses (Techspec DCX lenses, $f=10 \mathrm{~cm}$ ) placed between the mirror and a prism (Schott, LaSFN9, $\left.n_{658}=1.85\right)$ in an $\mathrm{f} \rightarrow 2 \mathrm{f} \rightarrow \mathrm{f}$ arrangement to minimize the movement of the laser spot on the base of the prism where the beam is subsequently reflected. After passing through another system of two lenses equivalent to the first one, the beam is focused on a photodiode detector (silicon photodiode, $>17 \mathrm{MHz} \mathrm{BW}, \lambda=350-1100 \mathrm{~nm}$ ). The silicon PIN photodiode (Thorlabs, FDS100) was reverse biased at $9 \mathrm{~V}$. A $1 \mathrm{k} \Omega$ resistor converts the induced photocurrent into a photovoltage. The data were collected using a datalogger (Omega, DaqPro-5300). For observation of the photokinetics, the reflectivity was monitored at the angle of steepest slope of the resonance curve (cf. Figure 3).

SPR Flow Cell. The flow cell was adapted from a design provided by W. Knoll and F. Yu (Max-Planck-Institute of Polymer Research, Mainz, Germany). ${ }^{42}$ A schematic is shown in Figure 2b. The gold-coated glass substrate (Schott, LaSFN9, $n=1.85$ ) was contacted with the prism through a film of index matching oil. Together, the quartz substrate, a $0.5 \mathrm{~mm}$ thick silicon spacer with a $7 \mathrm{~mm} \times 9 \mathrm{~mm}$ elliptical hole, and a quartz window with two steel capillaries made up the flow cell which was connected to a peristaltic pump (Ismatec, flow rate of $0.5 \mathrm{~mL} / \mathrm{min}$ ) with Tygon tubing.

Illumination of SPR Samples. For the illumination of the adlayer on the gold surface, the fiber tip of the illumination equipment described above was brought into direct contact with

(42) Yu, F.; Yao, D.; Knoll, W. Anal. Chem. 2003, 75, 2610-2617.
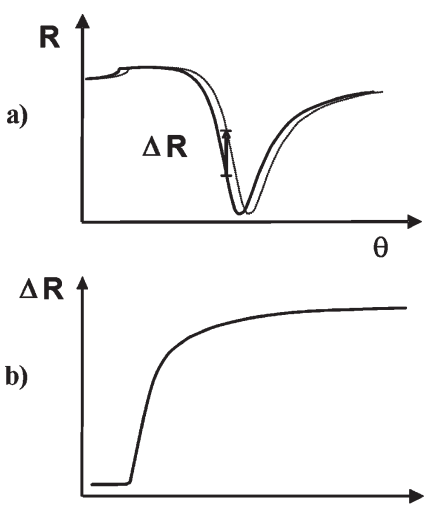

Figure 3. Principle of SPR signal detection at a fixed angle. (a) SPR-related angular dependence of reflectivity. (b) Timedependent signal change at a fixed angle.

the quartz window of the SPR cell. The radius of the illuminated spot on the gold film was $3.0 \mathrm{~mm}$, corresponding to an illuminated area of $0.28 \mathrm{~cm}^{2}$ with a corresponding photon fluence of $3.6 F_{\mathrm{ph}} \mathrm{cm}^{-2}$, where $F_{\mathrm{ph}}$ is the total photon flux from the fiber tip. In the quantitative photokinetic experiments at $365 \mathrm{~nm}$, $F_{\text {ph }}$ amounted to $7.0 \times 10^{-8} \mathrm{E} \mathrm{s}^{-1}$.

Monolayer Preparation. The glass substrates were cleaned in a 2\% Hellmanex solution, Millipore water, and absolute ethanol and dried in a stream of nitrogen. After the evaporation of $50 \mathrm{~nm}$ of gold (Allgemeine Gold-und Silberscheideanstalt AG, 99.99\% pure) in a vacuum chamber at a pressure of $<2 \times 10^{-6}$ mbar with a rate of $0.5 \mathrm{~nm} \mathrm{~s}^{-1}$, the substrates were immediately exposed to the $1 \mathrm{mM}$ ethanolic thiol solutions. They remained in the liquid overnight. After removal, they were washed repeatedly with ethanol and dried in a stream of nitrogen.

\section{Results}

The 2-(2-nitrophenyl)propoxycarbonyl (NPPOC) moiety has been well-established as a highly efficient photolabile protecting group in DNA chip synthesis. ${ }^{43-45}$ The mechanism of its photocleavage proceeds through a $\beta$-elimination and decarboxylation reaction following the primary intramolecular transfer of the benzylic hydrogen atom to the nitro group (cf. Figure 1). The photochemical quantum yield of this reaction is on the order of 0.5 . $^{46}$ To investigate the reaction on gold surfaces, an $n$-hexyl thiol anchor was attached to the NPPOC-protected thymidine through a succinic acid diester linkage at the $3^{\prime}-\mathrm{OH}$ position. In addition to the compound protected by the parent NPPOC group, the compound with the 5-substituted iodine derivative of NPPOC was also synthesized. For one thing, the iodine substituent increases the absorption coefficient ${ }^{47}$ and therefore yields an increased light sensitivity; second, it represents a specific and sensitive label for XPS analysis.

3.1. XPS Experiments. SAMs of compound 2 were prepared as described in Experimental Section on $5 \mathrm{~mm} \times 10 \mathrm{~mm}$ substrates cut from gold-coated silicon wafers. They were subjected to XPS measurements after being dried in a stream of nitrogen. For induction of the photocleavage reaction of the

(43) Hasan, A.; Stengele, K.-P.; Giegrich, H.; Cornwell, P.; Isham, K. R.; Sachleben, R. A. Pfleiderer, W ; Foote, R. S. Tetrahedron 1997, 53, 4247-4264.

(44) Giegrich, H.; Eisele-Bühler, S.; Hermann, C.; Kvasyuk, E.; Charubala, R.; W., P. Nucleosides Nucleotides 1998, 17, 1987-1996.

(45) Bühler, S.; Lagoja, I.; Giegrich, H.; Stengele, K.-P.; Pfleiderer, W. Helv. Chim. Acta 2004, 87, 620-659.

(46) Walbert, S.; Pfleiderer, W.; Steiner, U. E. Helv. Chim. Acta 2001, 84, 16011611

(47) At $365 \mathrm{~nm}$, the mercury line usually employed for effecting the photocleavage of the group, $\varepsilon=274 \mathrm{M}^{-1} \mathrm{~cm}^{-1}$ for NPPOC but $873 \mathrm{M}^{-1} \mathrm{~cm}^{-1}$ for I-NPPOC (values in EtOH). 


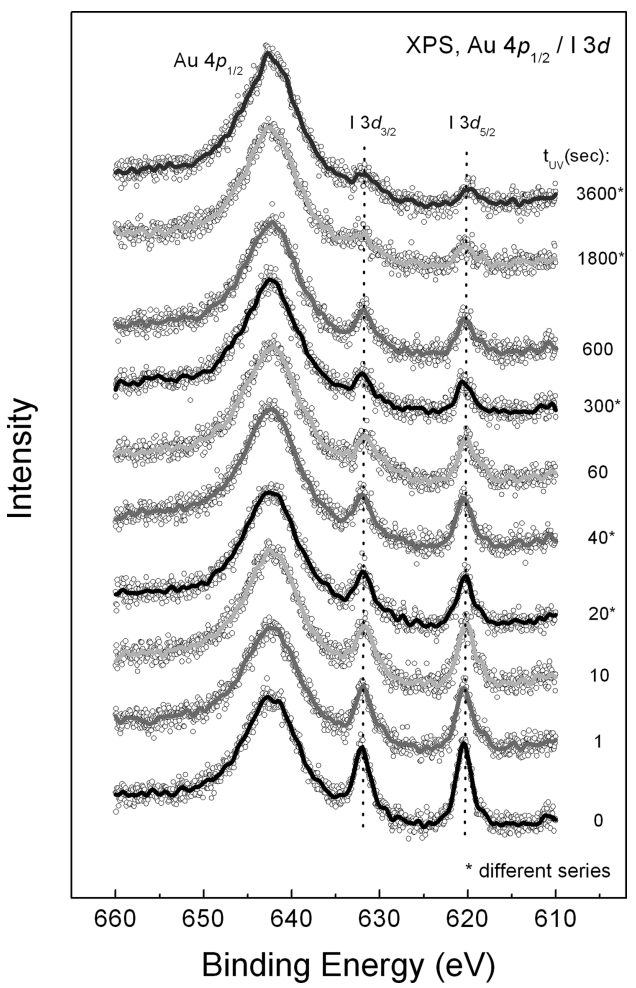

Figure 4. Detail of the XPS scan showing the iodine $3 \mathrm{~d}$ doublet at $620 / 632 \mathrm{eV}$ and the gold $4 \mathrm{p}_{1 / 2}$ signal at $643 \mathrm{eV}$ for different irradiation times of SAMs of $\mathbf{2}$.

I-NPPOC group in the SAM, the samples were irradiated for certain periods of time with $365 \mathrm{~nm}$ light from a fiber source at an irradiance of $\sim 0.5-1 \times 10^{-7} \mathrm{E} \mathrm{cm}^{-2} \mathrm{~s}^{-1}$. During irradiation, the substrate was rinsed with a continuous stream of absolute ethanol flowing from an injection needle at a rate of $0.5 \mathrm{~mL} / \mathrm{min}$. After irradiation, the sample was dried in a stream of nitrogen and analyzed by XPS. The high-resolution spectra covering the region of the iodine $3 \mathrm{~d}$ doublet at $620 / 632 \mathrm{eV}$ are shown in Figure $4 .^{48}$ Whereas the intensity of the sulfur signal does not change during irradiation (cf. Figure S5 of the Supporting Information), there is a systematic decrease in the iodine peaks. Control experiments with SAMs for the photounreactive reference compound $\mathbf{3}$ did not reveal any change in the iodine peak during irradiation for $1800 \mathrm{~s}$ under the same conditions (cf. Figure S4 of the Supporting Information)

The time dependence of the iodine peak intensity is shown in Figure 5. Loss of the iodine signal must be attributed to the photocleavage and release of the I-NPPOC group from the surface. The kinetics is characterized by a faster stage and a slower stage, each of them covering $\sim 50 \%$ of the signal decay. The faster stage is completed after irradiation for $\sim 60 \mathrm{~s}$. Fitting the kinetic decay by a sum of two exponentials yields decay constants of 12 and $4000 \mathrm{~s}$.

3.2. SPR Experiments. The SAM formation of compound 2 on gold and its behavior under photoillumination were directly monitored by SPR. Figure 6 presents a sensorgram of an experiment in which compound $\mathbf{2}$ was adsorbed on a freshly evaporated

(48) A survey spectrum displaying the specific lines of gold as the main features is shown in Figure S2 of the Supporting Information. Less prominent peaks due to carbon and oxygen are also assigned. Signals of the marker atom iodine are just faintly perceptible in the survey spectrum but are detectable with a good signal-tonoise ratio at higher resolution (cf. Figures S3 and S4 of the Supporting Information that also document the good reproducibility of the iodine signal intensity relative to that of the neighboring gold peak). gold film from a $1 \mathrm{mM}$ solution in ethanol passing through the flow cell (cf. Experimental Section). The formation of an adlayer is indicated by a rising SPR signal approaching saturation at $\sim 1500$ s (point A). After the flow had been switched to pure ethanol for the removal of unspecifically adsorbed 2, the SPR signal shows partial reversion to $\sim 80 \%$ of the maximum SPR signal level. This change is due to the removal of unspecifically adsorbed material. Next (cf. point B in Figure 6), the SPR sample is illuminated with light of the mercury line at $438 \mathrm{~nm}$ which is clearly outside the spectral region absorbed by compound $\mathbf{2}$ (for an absorption spectrum, cf. Figure S1 of the Supporting Information). During each illumination period, the SPR signal exhibits a downward peak, which, however, is fully and immediately reversed in the subsequent dark period. Actually, the "peaks" appearing in the diagrams of Figures 6 and 7, where the illumination periods are short in comparison to the overall time period recorded, are of constant intensity throughout the illumination time. This is shown more clearly in Figure 8 (cf. below). The same type of signal is obtained if bare gold films are illuminated, whether at 438 or $365 \mathrm{~nm}$. (cf. Figure S6 of the Supporting Information). We suppose that in this case the lightinduced signal peaks are due to unspecific heating effects. It was found that this change is due to a shift of the surface plasmon resonance curve to a smaller angle and not to a broadening of this resonance curve. After the irradiation wavelength had been switched to the mercury line at $365 \mathrm{~nm}$ (point $\mathrm{C}$ in the upper sensorgram of Figure 6), irreversible signal changes remain after reversion of the unspecific heating peaks. These irreversible changes are indicative of photoremoval of the protecting groups.

When SAMs of the nonphotolabile compound $\mathbf{3}$ are illuminated, no permanent SPR signal change with $365 \mathrm{~nm}$ light is observed (cf. Figure S6 of the Supporting Information), which is in line with the results obtained by XPS. Altogether, these observations confirm that unless the SAMs include photocleavable components there is no permanent change in the SPR signal under the illumination conditions of our experiments.

The photoinduced signal changes obtained when the photolabile compound is adsorbed during $\sim 30$ min directly in the flow cell are comparatively weak. Much more pronounced photoeffects were obtained when the gold-coated substrate was immersed in a solution of $\mathbf{1}$ for $\sim 15 \mathrm{~h}$ and the flow cell was assembled with the SAM already bound to the gold (cf. Experimental Section). A representative SPR signal trace for illumination first at $438 \mathrm{~nm}$ and then at $365 \mathrm{~nm}$ is included in Figure 6. Under illumination at $365 \mathrm{~nm}$, pronounced signal steps evolve. It must be mentioned, though, that the signal changes are partially developed during the dark phases of the illumination series. As a possible reason, a slow physical desorption kinetics of the detached photoproducts must be considered. Results of experiments shown in Figure 7 lend support to this hypothesis. Here we compare an SPR signal trace for a SAM prepared by immersion of the substrate in a solution of 2 for some $15 \mathrm{~h}$ with another signal trace for a sample prepared from a solution containing mercaptohexanol as a competitor for the binding to the gold. Thus, a mixed SAM with a reduced content of $\mathbf{2}$ should be formed. As shown in Figure 7, for such a SAM most of the photoeffect is developed immediately during the stage of illumination.

To determine the photokinetics over a longer period of illumination in a similar manner as recorded by XPS measurements (cf. Figure 5), we performed a corresponding long-term SPR experiment. SPR signal changes induced by the illumination with $365 \mathrm{~nm}$ light were observed for an overall illumination period of $1 \mathrm{~h}$ (cf. Figure 8). For the longer illumination periods after $\sim 2500 \mathrm{~s}$, we clearly observed that when the illumination is 


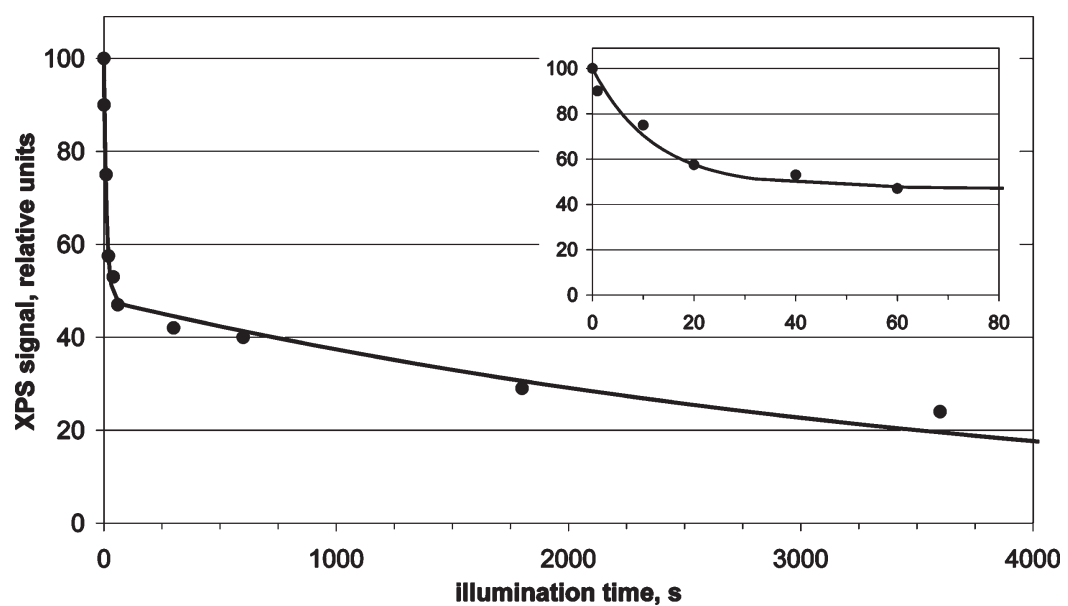

Figure 5. Photokinetics of decay of the XPS peak of iodine upon irradiation of SAMs of $\mathbf{2}$ on gold. The inset shows an expansion of shorttime behavior.

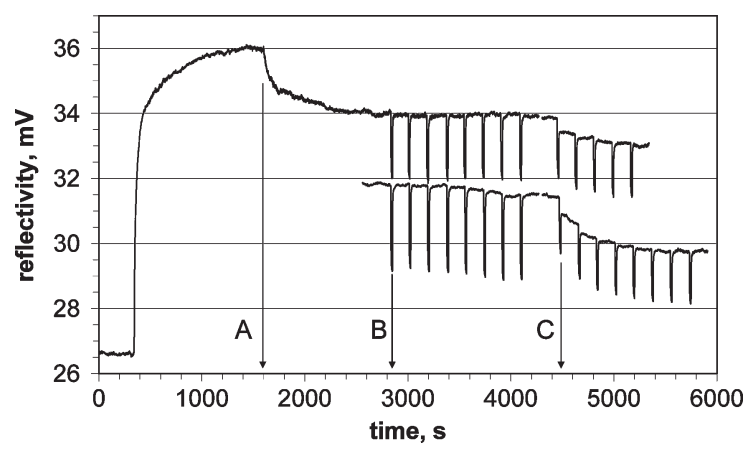

Figure 6. SPR sensorgram (top trace) recorded for a $1 \mathrm{mM}$ solution of compound $\mathbf{2}$ in ethanol. The flow rate through the SPR cell amounted to $0.5 \mathrm{~mL} / \mathrm{min}$. At point $\mathrm{A}$, the flow was switched to the pure solvent. Point B marks the start of eight periods of illumination for $15 \mathrm{~s}$ with $438 \mathrm{~nm}$ light and a $150 \mathrm{~s}$ dark interval. From point $\mathrm{C}$, the illumination was performed with $365 \mathrm{~nm}$ light. SPR sensorgram (bottom trace) for a SAM of compound 1 prepared overnight. During the observation in the SPR cell, the substrate is purged with ethanol at a rate of $0.5 \mathrm{~mL} / \mathrm{min}$. From time B to C, the sample was illuminated with $438 \mathrm{~nm}$ light and from time $\mathrm{C}$ with $365 \mathrm{~nm}$ light.

switched on, the SPR signal amplitude is shifted by a constant value. The signal drift during the illumination is due to the photoreaction. After the light is switched off, the SPR signal jumps back by the same amount as when it is switched on. During the dark periods, the SPR signal is constant, except for the early parts of the signal trace, where some indications of delayed desorption appear. As in the case of the XPS-detected kinetics, the SPR-detected photokinetics also consist of a faster and a slower stage (cf. Figure 9). The faster stage is finished after $\sim 60 \mathrm{~s}$. Fitting the kinetic decay by a sum of two exponentials yields decay constants of 16 and $1100 \mathrm{~s}$.

\section{Discussion}

The XPS and SPR experiments have clearly shown NPPOCspecific photocleavage to occur in SAMs of $\mathbf{2}$ on gold. As the comparison with the behavior of compound $\mathbf{3}$ has demonstrated, the disappearance of the iodine signal in the XPS spectrum is not due to a direct cleavage of the carbon-iodine bond. Thus, the very similar photokinetics of the disappearance of the XPS iodine peaks and the change in the SPR signal during photoillumination of $\mathbf{2}$ on gold provides convincing evidence that the change in the
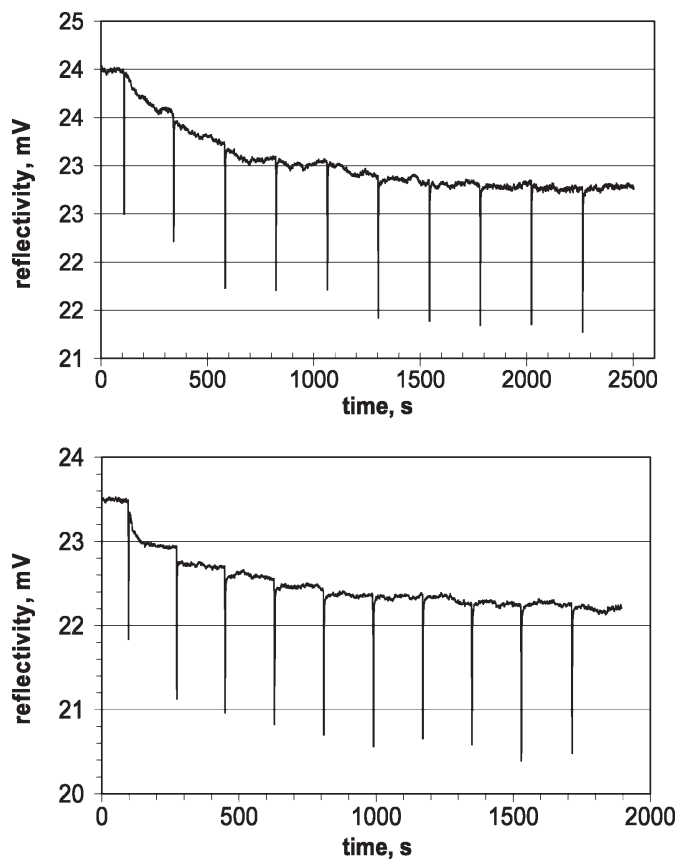

Figure 7. SPR sensorgram for a SAM of compound 2 prepared overnight (top). The SAM was illuminated 10 times for $2 \mathrm{~s}$ with 365 $\mathrm{nm}$ light with a constant ethanol flow. SPR sensorgram for a mixed SAM of compound $\mathbf{2}$ and mercaptohexanol prepared by soaking for $\sim 15 \mathrm{~h}$ in a solution containing $0.6 \mathrm{mM} 2$ and $0.33 \mathrm{mM}$ mercaptohexanol (bottom). The SAM was illuminated 10 times for $2 \mathrm{~s}$ with $365 \mathrm{~nm}$ light under a constant flow of ethanol.

SPR signal reflects the same photoreaction. The overall change in the SPR signal voltage during photocleavage of $\mathbf{2}$ corresponds to 4-5 $\mathrm{mV}$ (cf. Figure 8). Following the method described by Campbell and co-workers, ${ }^{15}$ we will now estimate the surface density of I-NPPOC groups corresponding to such a signal amplitude and will compare it to the density of a hypothetical layer of I-NPPOCOH.

Formally, we are dealing with a four-layer system (cf. Figure 10) consisting of the sandwiching gold and solvent layers and the SAM-type adlayer which we divide into two sublayers, one constituted by the linker chains with the attached nucleoside (thickness $d_{\mathrm{b}}$ ) and one constituted by the photoreleasable I-NPPOC groups (thickness $d_{\mathrm{a}}$ ). In a strict geometric sense, the 
boundary between these sublayers is not sharp. The nucleoside extends into both. Hence, the parameter $d_{\mathrm{a}}$ truly refers to the

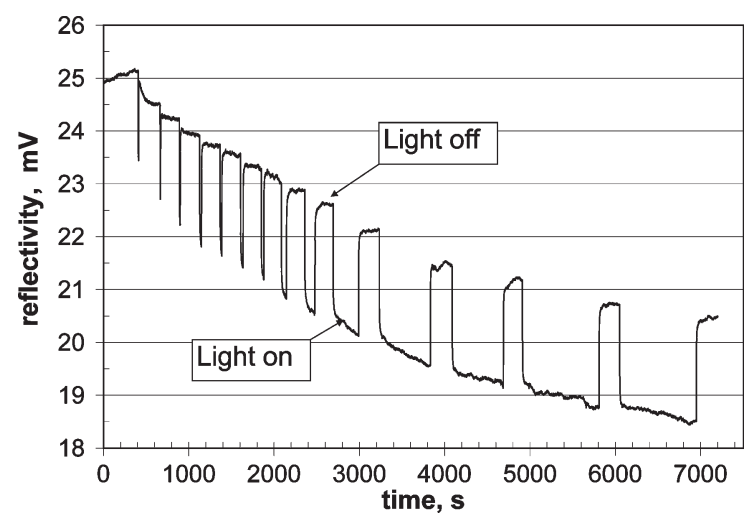

Figure 8. SPR sensorgram for a SAM of compound 2 prepared by immersion of the gold-coated substrate into a $1 \mathrm{mM}$ ethanolic solution of 2 for $\sim 15 \mathrm{~h}$. The SAM was illuminated with $365 \mathrm{~nm}$ light for $1 \mathrm{~h}$ altogether in different intervals $(2 \times 5 \mathrm{~s}, 10 \mathrm{~s}, 2 \times 20 \mathrm{~s}$, $2 \times 30 \mathrm{~s}, 60 \mathrm{~s}, 120 \mathrm{~s}, 300 \mathrm{~s}, 2 \times 600 \mathrm{~s}$, and $2 \times 900 \mathrm{~s}$ ) during and between which a constant flow of ethanol was maintained. thickness of a fictitious equivalent layer, exclusively filled by the I-NPPOC group. Following ref 15, the change in reflectivity on replacement of layer a by the solvent can be expressed as

$$
\Delta R_{\mathrm{a}}=m\left(n_{\mathrm{s}}-n_{\mathrm{a}}\right)\left[1-\exp \left(-2 d_{\mathrm{a}} / l_{\mathrm{d}}\right)\right] \exp \left(-2 d_{\mathrm{b}} / l_{\mathrm{d}}\right)
$$

where $m$ is the sensitivity parameter (in our experiment $m=3400$ $\mathrm{mV} / \mathrm{RIU}$; cf. Experimental Section for the calibration method), $n_{\mathrm{a}}$ and $n_{\mathrm{s}}$ are the refractive indices of layers a and $\mathrm{b}$, respectively, and $l_{\mathrm{d}}$ is the decay length of the evanescent field above the gold surface. With the approximation ${ }^{15} l_{\mathrm{d}} \approx 0.37 \lambda$, where $\lambda$ is the laser wavelength, we obtain an $l_{\mathrm{d}}$ of $\approx 243 \mathrm{~nm}$. Since $d_{\mathrm{a}}$ and $d_{\mathrm{b}}$ are much smaller than $l_{\mathrm{d}}$, we can derive a linear approximation to the expression for $\Delta R_{\mathrm{a}}$ in eq 1 :

$$
\Delta R_{\mathrm{a}} \approx m\left(n_{\mathrm{s}}-n_{\mathrm{a}}\right) \frac{2}{l_{\mathrm{d}}} d_{\mathrm{a}} f_{\mathrm{s}}
$$

Here we also introduced a fractional filling factor $0 \leq f_{\mathrm{s}} \leq 1$ quantitating the degree of replacement of I-NPPOC groups by the solvent if only part of these groups has undergone photocleavage. The refractive index $n_{\mathrm{a}}$ of the I-NPPOC layer must be estimated

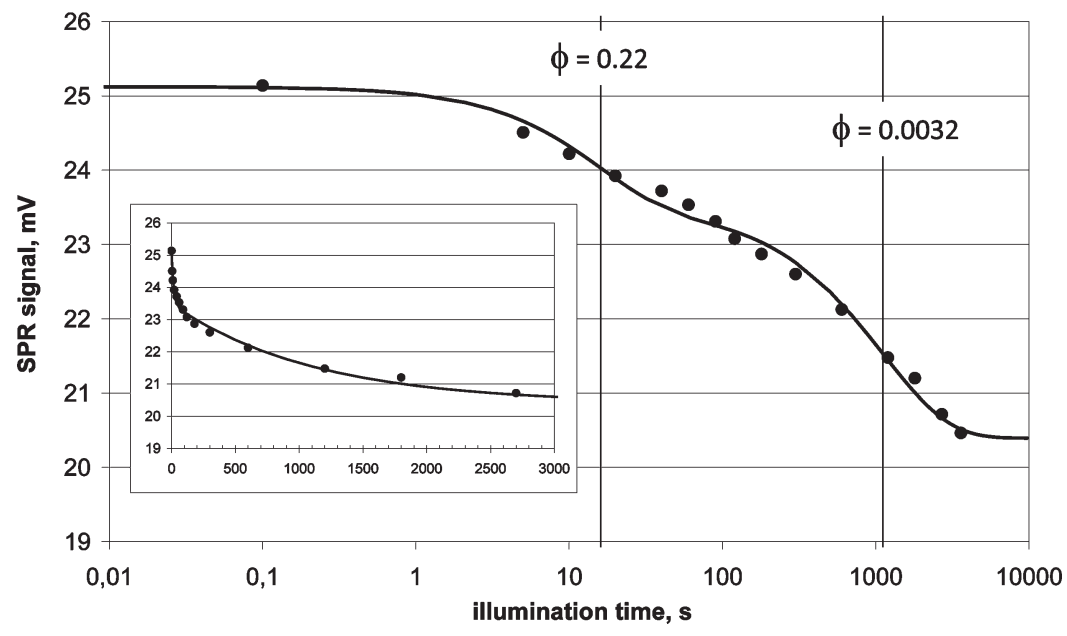

Figure 9. Photokinetics deduced from the SPR signal in Figure 8, on a logarithmic time scale. The vertical lines mark the time constants used for the biexponential fit and the equivalent quantum yields (cf. Discussion). The inset has a linear time scale.

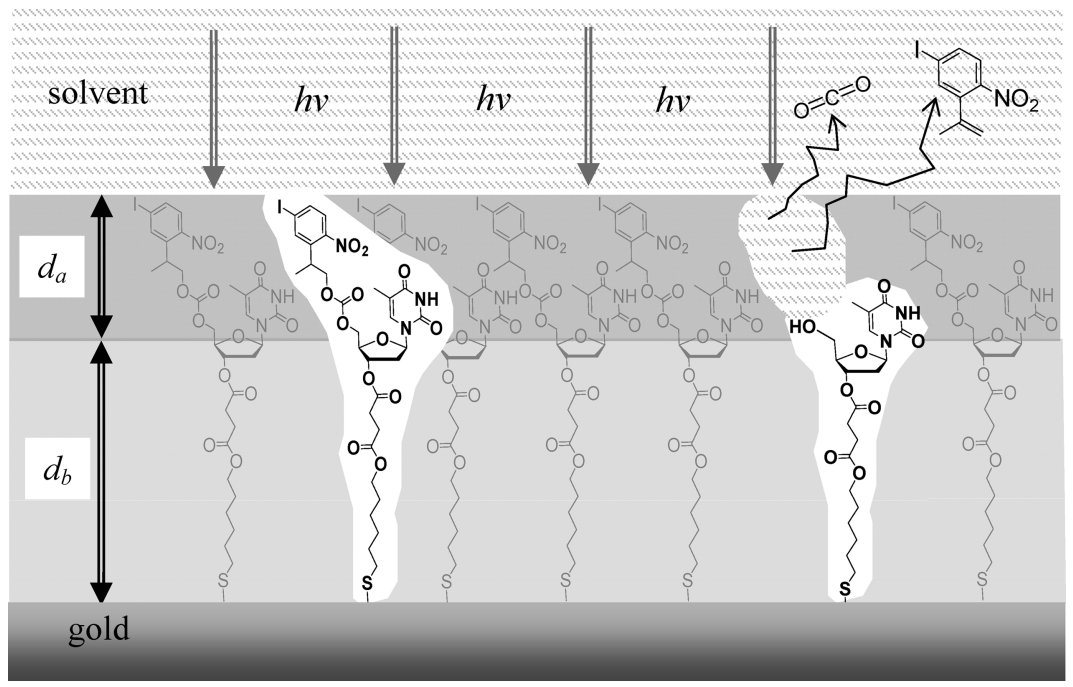

Figure 10. Four-layer model (gold, linkers, headgroups, and solvent) for analyzing the SPR effect of SAMs of compound 2 on gold. After photorelease of the I-NPPOC group, the void is filled by the solvent. Although the nucleobase and the protecting group cannot be sharply assigned to different layers, formally, layer a is assigned to the I-NPPOC group only. 
by combining refractive index values and molar volumes that can be assigned to parts of the structure on the basis of the additivity rule for the Lorentz-Lorenz molar refraction $R_{\mathrm{m}}$

$$
R_{\mathrm{m}}=V_{\mathrm{m}} \frac{n^{2}-1}{n^{2}+2}
$$

Combining the refractive indices and molar volumes of benzene (1.50 and $26.2 \mathrm{~mL}$, respectively), iodobenzene (1.62 and $112 \mathrm{~mL}$, respectively), and nitrobenzene (1.55 and $94.4 \mathrm{~mL}$, respectively), we obtain an $n$ of $\approx 1.65$ and a $V_{\mathrm{m}}$ of $\approx 121 \mathrm{~mL}$ for the iodonitrobenzene fragment. The refractive properties of the propoxycarbonyl group are comparable to those of ethyl acetate $(n=$ 1.37; $V_{\mathrm{m}}=98 \mathrm{~mL}$ ). Combining both fragments yields an $n$ of $\approx 1.52$ and a $V_{\mathrm{m}}$ of $\approx 219 \mathrm{~mL}$ for a condensed I-NPPOCOH layer. Thus, for a complete $\left(f_{\mathrm{s}}=1\right)$ replacement of the I-NPPOC layer by the solvent ethanol $(n=1.36)$, we expect $\Delta R_{\mathrm{a}} / d_{\mathrm{a}} \approx 4.5$ $\mathrm{mV} / \mathrm{nm}$. Comparing this result with an observed $\Delta R$ of $4-5 \mathrm{mV}$ yields a $d_{\mathrm{a}}$ of $\approx 1.0 \mathrm{~nm}$ for the layer characterized by the refractivity index of the photolabile I-NPPOC groups. Since the length of the photolabile groups (cf. Figure S7 of the Supporting Information) corresponds to just $\sim 1 \mathrm{~nm}$, layer a is consistently represented by a densely packed monomolecular layer of parallel aligned I-NPPOC groups.

The analysis of the observed photokinetics must be based on the rate law

$$
\dot{\sigma}=-k_{\mathrm{abs}} \phi \sigma
$$

where $\sigma$ is the surface density of the NPPOC group, $k_{\text {abs }}$ is the effective rate constant for absorbing a photon at the incident wavelength, and $\phi$ is the quantum yield of photocleavage. The rate constant $k_{\text {abs }}$ is related to the extinction coefficient $\varepsilon_{\text {eff }}$ and the photon flux density $I$ at the position of the photoabsorbing molecule by

$$
k_{\mathrm{abs}}=(\ln 10) \varepsilon_{\mathrm{eff}} I
$$

The effective absorption coefficient $\varepsilon_{\text {eff }}$ depends on the orientational distribution of the transition dipole moment. ${ }^{49}$ If this distribution is isotropic, $\varepsilon_{\text {eff }}$ equals the normal (isotropic) absorption coefficient $\varepsilon_{\text {iso }}$. Integrating eq 4 by assuming that all NPPOC groups have sufficient rotational mobility to maintain the isotropic distribution even though the photoexcitation is orientationally selective leads to a monoexponential time dependence

$$
\sigma(t)=\sigma_{0} \exp \left[-(\ln 10) \varepsilon_{\text {iso }} \phi I t\right]
$$

On the other hand, if we assume that the orientations of the NPPOC groups are fixed, an orientation-dependent value of $\varepsilon$ must be used. Denoting the angle between the direction of the light (i.e., the normal to the surface) and the transition dipole moment by $\vartheta$, one has

$$
\varepsilon(\vartheta)=3(\cos \vartheta)^{2} \times \varepsilon_{\text {iso }}
$$

Averaging eq 6 with an orientation-dependent $\varepsilon$ over an isotropic distribution of angles $\vartheta$ yields

$$
\sigma(t)=\frac{\sqrt{\pi}}{2} \sigma_{0} \frac{\operatorname{erf}\left[\sqrt{3(\ln 10) \varepsilon_{\text {iso }} \phi I t}\right]}{\sqrt{3(\ln 10) \varepsilon_{\text {iso }} \phi I t}}
$$

Such a function would indeed reproduce the observed photokinetic behavior in a qualitative manner (Figure 11a). However,

(49) Labhart, H. Tetrahedron 1963, 19(Suppl. 2), 223-241.
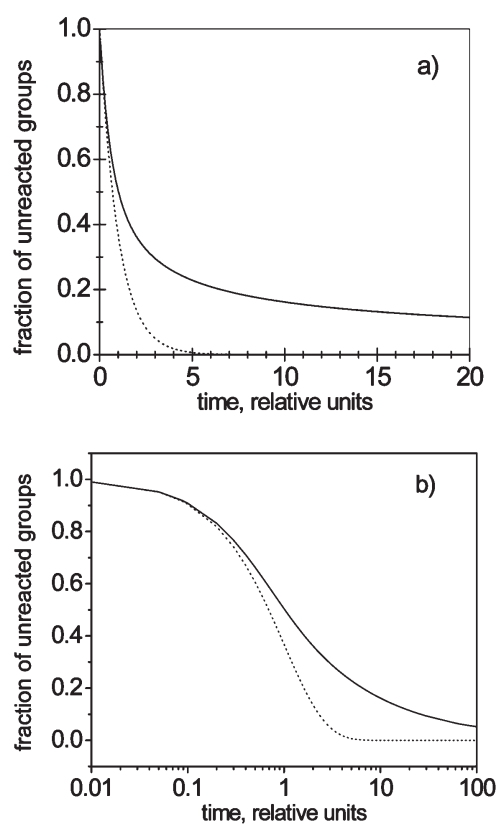

Figure 11. Theoretical photokinetic curves for rotationally mobile NPPOC groups with an isotropic $\varepsilon$ (dashed, eq 6) and for orientationally fixed NPPOC groups with an isotropic orientational distribution (solid line, eq 8): (a) linear time scale and (b) log time scale.

\section{Scheme 1}
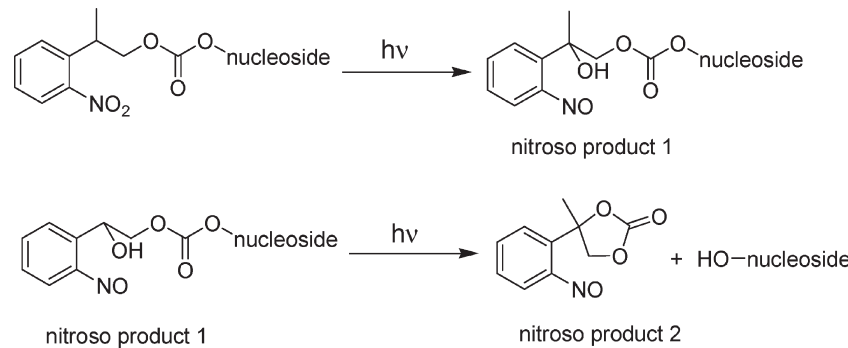

as is clearly displayed in a log plot (Figure 11b), the shape of the decay function corresponds to a stretched exponential rather than to a biexponential function as suggested by the observed behavior (cf. Figure 9). Furthermore, it is not very likely that the NPPOC groups in the SAM are orientationally fixed to such an extent as required to make the assumptions leading to eq 8 valid.

A more realistic interpretation of the biphasic photokinetic behavior should consider some inhomogeneity in the photochemical quantum yield $\phi$. Presently, we are aware of two possibilities:

(i) It is well-known that, depending on the basicity of the solvent, the side reactions shown in Scheme 1 may participate significantly: $:^{9,46}$ In the first of these reactions, there is no release of the protecting group, but there is a possibility of such a release in a subsequent reaction ( $\mathrm{cf}$. the second reaction in Scheme 1). ${ }^{9}$ While in homogeneous solution the quantum yield of the $\beta$-elimination reaction (cf. Figure 1) and of the photo-oxygenation of the benzylic carbon (first reaction in Scheme 1) may reach the same order of magnitude, ${ }^{46}$ the quantum yield of the second reaction in Scheme 1 and its solvent dependence have not been investigated in detail. Very likely, this quantum yield is much lower than the former. In preliminary SPR-monitored photokinetic experiments, we found that addition of sodium acetate as a base has a strong effect on the amplitude of the slow stage of the photokinetics. As 
in homogeneous solution, the presence of proton acceptors would appear to favor the $\beta$-elimination pathway (Figure 1) over the nitroso pathway (first reaction in Scheme 1). This observation represents a strong argument in favor of suggestion (i).

(ii) It is not known whether all the NPPOC groups are stretched away from the gold surface as shown in Figure 10, and it is wellknown that the level of quenching of electronically excited states due to the transfer of energy to the metal strongly increases as a molecule moves closer to the metal surface. ${ }^{17}$ Quantitative data on quantum yield dependences on the metal-substrate distance are, however, rare. For photochemical trans-cis isomerization of azobenzene carrying a polymethylenethiol linker, it was reported by Fox and co-workers ${ }^{31}$ that the quantum yield on gold colloid particles is strongly reduced in comparison to the quantum yield in homogeneous solution, and there was a clear dependence of this effect on the length of the polymethylene linker. Thus, for a chain of four methylene groups, the quantum yield was reduced by a factor of 137 , whereas for 12 methylene groups, it was a factor of only 1.5 .

With knowledge of the values of $I$ and $\varepsilon_{\text {eff }}$, pertinent photochemical quantum yields can be calculated from the time constants given in relation to Figures 5 and 9 . With regard to the value of $I$, we note that in front of the gold surface the value experienced by the molecules differs from that determined by actinometry in solution because of the reflected light and its interference with the incoming light. For a $50 \mathrm{~nm}$ gold layer $\left(n_{365}=1.715 ; \kappa_{365}=\right.$ $1.863)^{50}$ between a highly refractive LaSFN9 glass $\left(n_{365}=1.923\right)$ and ethanol $(n=1.36)$, we estimate ${ }^{51,52}$ a reflectivity $(R)$ of 0.30 and a phase shift between incoming and reflected light of -2.31 $\left(-132^{\circ}\right)$. Due to partial cancellation of the electric fields of incoming and reflected light, the effective intensity experienced by the molecules in front of the gold surface is only $57 \%$ of that of the incoming light.

Using an isotropic $\varepsilon_{365}$ (I-NPPOC) value of $873 \mathrm{M}^{-1} \mathrm{~cm}^{-1}$ and the actinometric light intensity corrected by the interference effect with the reflected light, we can assign quantum yield values of 0.22

(50) Palik, E. D. Handbook of Optical Constants of Solids; Academic Press: San Diego, 1998

(51) Yeh, P. Optical waves in layered media; John Wiley \& Sons: New York, 1988.

(52) A useful Internet tool for online calculation of reflectivity/transmissivity for light incident at an arbitrary angle on a thin film stack is provided by G. Boisset. The link is www.luxpop.com. and 0.0032 to the two kinetic stages determined in the SPR experiment (cf. Figure 9). The short time value of 0.22 is similar in terms of the order of magnitude as observed in a homogeneous solution ( 0.40 in $\left.\mathrm{MeOH} / \mathrm{H}_{2} \mathrm{O}, 1: 1, \mathrm{v}: \mathrm{v}\right),{ }^{53}$ indicating little quenching of the photoreactive electronic state at least for $\sim 50 \%$ of all the I-NPPOC groups.

\section{Conclusions}

In this work, we have demonstrated that the kinetics of photorelease of photolabile NPPOC groups from a protected nucleoside, linked by a mercaptoalkyl anchor to a gold surface, can be directly monitored by SPR. An iodine substituent as a marker for XPS has proven to be advantageous in verifying the chemistry responsible for the change in the SPR signal. These results represent a first step toward a complete online monitoring of the photolithographic synthesis of DNA chips. Spatial resolution was not an issue in our work. Next steps should explore the possibilities of light-addressed photocleavage on NPPOC-functionalized SAMs with parallel multispot detection in SPR microscopic mode, ${ }^{54}$ demonstrated so far in various applications with lateral resolutions of $\sim 200 \mu \mathrm{m} \times 200 \mu \mathrm{m}^{55}$ or even less. ${ }^{56}$

Acknowledgment. This work was conducted within Research Unit 434 (Oligosaccharide and DNA chips, recognition of secondary gene products) and collaborative research center SFB 767 funded by the Deutsche Forschungsgemeinschaft (DFG Ste 283/7). Practical advice on the design of the SPR cell from Prof. Dr. W. Knoll and Dr. F. Yu (Max-Planck-Institute of Polymer Research) is gratefully acknowledged.

Supporting Information Available: UV-vis spectra of compounds 1 and 2 (1.), XPS spectra (2.), SPR signals (3.), geometry of the I-NPPOC group (4.), and synthesis (5.). This material is available free of charge via the Internet at http:// pubs.acs.org.

(53) Walbert, S. Mechanistische Untersuchungen zur Abspaltung photolabiler Schutzgruppen. Doctoral Dissertation, University of Konstanz, Konstanz, Germany, 2003.

(54) Rothenhäusler, B.; Knoll, W. Nature 1988, 332, 615-617.

(55) Shumaker-Parry, J. S.; Campbell, C. T. Anal. Chem. 2004, 76, 907-917.

(56) Hickel, W.; Knoll, W. J. Appl. Phys. 1990, 67, 3572-3575. 